\title{
Artemisia annua Leaf Extract Attenuates Hepatic Steatosis and Inflammation in High-Fat Diet-Fed Mice
}

\author{
Kyung Eun Kim, ${ }^{1, *}$ Keon-Hee Ko, ${ }^{2,3, *}$ Rok Won Heo, Chin-ok Yi, ${ }^{1}$ Hyun Joo Shin, \\ Jun Young Kim, ${ }^{4}$ Jae-Ho Park, ${ }^{5}$ Sanghae Nam, ${ }^{3}$ Hwajin Kim, and Gu Seob Roh ${ }^{1}$ \\ ${ }^{1}$ Department of Anatomy and Convergence Medical Science, Institute of Health Sciences, Gyeongsang National \\ University School of Medicine, Gyeongnam, Republic of Korea. \\ ${ }^{2}$ Gyeongnam Oriental Medicinal Herb Institute, Gyeongnam, Republic of Korea. \\ ${ }^{3}$ Division of Food Science, Gyeongnam National University of Science and Technology, Gyeongnam, Republic of Korea. \\ ${ }^{4}$ Busan Regional Korea Food and Drug Administration, Ministry of Food and Drug Safety, Busan, Republic of Korea. \\ ${ }^{5}$ Shinseon F\&V Co., Ltd., Gyeongnam, Republic of Korea.
}

\begin{abstract}
Artemisia annua L. (AA) is a well-known source of the antimalarial drug artemisinin. AA also has an antibacterial and antioxidant activity. However, the effect of AA extract on hepatic steatosis induced by obesity is unclear. We investigated whether AA extract prevents obesity-induced insulin resistance and hepatic steatosis in high-fat diet (HFD)-fed mice. Mice were randomly divided into groups that received a normal chow diet or HFD with or without AA for 12 weeks. We found that AA extract reduced insulin resistance and hepatic steatosis in HFD-fed mice. Western blot analysis showed that HFD-induced expression of nuclear sterol regulatory element-binding protein 1 and carbohydrate-responsive element-binding protein in the livers was decreased by AA extract. In particular, dietary administration of AA extract decreased hepatic highmobility group box 1 and cyclooxygenase-2 expression in HFD-fed mice. AA extract also attenuated HFD-induced collagen deposition and fibrosis-related transforming growth factor- $\beta 1$ and connective tissue growth factor. These data indicate that dietary AA extract has beneficial effects on hepatic steatosis and inflammation in HFD-fed mice.
\end{abstract}

\section{KEY WORDS: • Artemisia annua L. $\bullet$ hepatic inflammation $\bullet$ hepatic steatosis $\bullet$ obesity}

\section{INTRODUCTION}

$\boldsymbol{A}$ RTEMISIA ANNUA L. (AA) has been used for many centuries in traditional Chinese medicine for the treatment of febrile diseases and malaria. ${ }^{1}$ AA contains numerous bioactive compounds. In particular, artemisinin and its derivatives have been clinically used to treat drug-resistant malaria. ${ }^{2}$ AA also possesses several bioactive functions, including anti-inflammatory activities that contribute to the therapeutic effects of the herb. ${ }^{3,4}$ However, the effect of AA on obesity-induced hepatic steatosis is largely unknown.

Obesity can cause a number of medical disorders, such as nonalcoholic fatty liver disease (NAFLD), metabolic syndrome, and type 2 diabetes. ${ }^{5}$ Excessive accumulation of

*These authors contributed equally to this study.

Manuscript received 31 May 2015. Revision accepted 25 October 2015.

Address correspondence to: Gu Seob Roh, MD, PhD, Department of Anatomy and Convergence Medical Science, Institute of Health Sciences, Gyeongsang National University School of Medicine, 15, 816 Beon-gil, Jinju-daero, Jinju, Gyeongnam 660-751, Republic of Korea, E-mail: anaroh@gnu.ac.kr triglycerides (TG) in hepatocytes is a hallmark of NAFLD. The spectrum of NAFLD can range from simple fatty liver (hepatic steatosis) with inflammation to the potentially progressive form of nonalcoholic steatohepatitis (NASH), which can progress to fibrosis and cirrhosis, resulting in increased morbidity and mortality. ${ }^{6,7}$ To control the development of NAFLD, determining the precise mechanism of how lipid accumulation is regulated in the liver, is important. ${ }^{8}$ Two key regulators of glucose metabolism and lipid synthesis in the liver are the carbohydrate-responsive element-binding protein (ChREBP) and sterol regulatory element-binding protein 1 (SREBP1). ${ }^{9}$ ChREBP, a glucoseresponsive transcription factor, regulates glycolysis and lipogenesis due to its transcriptional control of the key enzymes of L-type pyruvate kinase, acetyl-CoA carboxylase (ACC), and fatty acid synthase. ${ }^{10}$ SREBP1 mediates insulin signaling by activating the expression of lipogenic genes, such as ACC. ${ }^{11}$ SREBP1 is also transcriptionally activated in hepatic steatosis; however, the protective role of AA on hepatic ChREBP and SREBP1 function on the pathogenesis of NAFLD remains unclear. 
Chronic high-fat diet (HFD)-induced insulin resistance results in lipogenesis and increased release of free fatty acids, which can be taken in by hepatocytes, consequently leading to hepatic lipid accumulation and inflammation. ${ }^{12}$ High-mobility group box 1 (HMGB1) protein released by the damaged liver can lead to prolonged inflammatory and immune responses and influence the progression of NAFLD to fibrosis. ${ }^{13}$ Additional factors that promote the fibrotic progression include angiotensin II, transforming growth factor- $\beta 1$ (TGF- $\beta 1$ ), and connective tissue growth factor (CTGF). ${ }^{14}$

In this study, we showed that AA extract administration reduced HFD-induced obesity and investigated the underlying mechanisms of the AA protection against lipogenesis and fibrogenesis.

\section{MATERIALS AND METHODS}

Plant materials and preparation of $A A$ extract

AAs were collected at a farm in Sancheong-gun, South Korea, in June 2013. Only young leaves of AA were harvested and then allowed to air-dry for 3 days at room temperature to remove excess moisture. After air-drying, AA leaves were pulverized to a fine powder using a mill. Five different polar solvent extraction systems were used; the polar solvents were $\mathrm{CHCl}_{3}$, ethyl acetate, ethanol, $80 \%$ aqueous ethanol, and $\mathrm{H}_{2} \mathrm{O}$. The $80 \%$ aqueous ethanol extract was selected as a target source for isolating $\alpha$-glucosidase inhibitors due to its highest $\alpha$-glucosidase inhibitory effects. The pulverized leaves $(1 \mathrm{~g})$ were extracted with $20 \mathrm{~mL} 80 \%$ aqueous ethanol at $25^{\circ} \mathrm{C}$ for $24 \mathrm{~h}$ with continuous agitation on a mechanical shaker. The supernatant was centrifuged at $4500 \mathrm{~g}$ for $5 \mathrm{~min}$ and then filtered through a $0.22-\mu \mathrm{m}$ syringe filter before analysis. Aliquots were filtered and used.

Ultra performance liquid chromatography-quadrupole time-of-flight mass spectrometry parameters and nuclear magnetic resonance spectrometry

Chromatographic separation was performed on a $2.1 \times 100 \mathrm{~mm}, 1.7-\mu \mathrm{m}$ ACQUITY BEH C18 chromatography column. The column temperature was maintained at $35^{\circ} \mathrm{C}$, and the mobile phases A and B were deionized water with $0.1 \%$ formic acid and acetonitrile with $0.1 \%$ formic acid, respectively. The mass spectrometer was operated in a positive ion mode. $\mathrm{N}_{2}$ was used as the desolvation gas. The desolvation temperature was set to $350^{\circ} \mathrm{C}$ at a flow rate of $500 \mathrm{~L} / \mathrm{h}$, and the source temperature was $100^{\circ} \mathrm{C}$. The capillary and cone voltages were set to 2700 and $30 \mathrm{~V}$, respectively. The data were collected for each sample with a 0.25 -sec scan time and a $0.01-\mathrm{sec}$ interscan delay. Leucine-enkephalin was used as the reference compound $(\mathrm{m} / \mathrm{z}$ 556.2771 in the positive mode). One- and twodimensional nuclear magnetic resonance (NMR) data were obtained on a Bruker AM-500 spectrometer in $\mathrm{CDCl}_{3}$, dimethyl sulfoxide (DMSO)- $d_{6}$, and acetone- $d_{6}$. Electron ionization mass spectrometry was performed on a JEOL JMS-700 mass spectrometer. A Waters ACQUITY Q-TOF UPLC/MS system was coupled on-line with a photodiode array (PDA 2996; Waters, Milford, MA, USA) detector. Mass spectrometry control and spectral processing were carried out using MassLynx software (version 4.1; Waters). The analytes were separated on a Waters ACQUITY BEH C18 column $(1.7 \mu \mathrm{m}, 2.1 \times 100 \mathrm{~mm})$.

\section{$\alpha$-Glucosidase inhibition assay}

$\alpha$-Glucosidase inhibitory activities were evaluated according to the method described by Matsui et al. with some modifications. ${ }^{15}$ The reaction mixture contained $20 \mu \mathrm{L} \alpha$-glucosidase $(0.5 \mathrm{U} / \mathrm{mL}), 75 \mu \mathrm{L}$ p-nitrophenyl- $\alpha$-Dglucopyranoside $(2.4 \mathrm{mM}), 50 \mu \mathrm{L}$ sample, and $825 \mu \mathrm{L}$ $50 \mathrm{mM}$ potassium phosphate buffer $(\mathrm{pH} 6.8)$. The $\alpha$ glucosidase activity was determined spectrophotometrically at $405 \mathrm{~mm}$ using a multimicroplate reader, SpectraMax M5 (Molecular Device, Sunnyvale, CA, USA). Acarbose was used as a positive control for $\alpha$-glucosidase inhibitor. The concentration of the sample required to inhibit $\alpha$ glucosidase activity by $50 \%$ was defined as the $\mathrm{IC}_{50}$ value. The inhibition rate $(\%)$ was calculated as follows:

$\alpha-$ Glucosidase inhibition $(\%)=\left(1-\frac{\text { sample absorption }}{\text { control absorption }}\right) \times 100$

\section{Animals and the obesity model}

Three-week-old male C57BL/6J mice were purchased from Koatech (Pyeongtaek, South Korea) and maintained in the animal facility at the Gyeongsang National University. The experiments were performed in accordance with the National Institutes of Health Guidelines on the Use of Laboratory Animals (GNU-140212-M0008). The mice were individually housed with an alternating 12-h light-12-h dark cycle. Starting at 4 weeks of age, mice were randomly assigned to four groups ( $n=10$ mice per group). The mice were fed for 12 weeks with either an HFD (45\%; Research Diets, New Brunswick, NJ, USA) or a standard diet (normal diet, ND). Mice were additionally fed with $400 \mathrm{mg} / \mathrm{kg}$ of AA extract daily on either the ND or HFD. The mice were weighed four times monthly and immediately before the sacrifice at 15 weeks of age.

\section{Glucose tolerance test and insulin tolerance test}

For the glucose tolerance test (GTT), the mice were fasted overnight $(16 \mathrm{~h})$ before the test, and the insulin tolerance test (ITT) was performed on the mice at 2 p.m., as previously described. ${ }^{16}$

\section{Measurement of serum metabolic parameters}

For serum analysis, the mice ( $n=10$ mice per group) were intramuscularly anesthetized with Zoletil $(5 \mathrm{mg} / \mathrm{kg}$; Virbac Laboratories, Carros, France). Serum aspartate aminotransferase (AST), alanine aminotransferase (ALT), and total cholesterol levels were determined in Green Cross Reference Laboratory (Yongin, South Korea). Serum insulin was measured using mouse insulin (Shibayagi Co., Gunma, Japan) enzyme-linked immunosorbent assay kit according to the manufacturer's instruction. 


\section{Hepatic TG colorimetric assay}

Frozen livers were homogenized and centrifuged, and the supernatants were used to determine TG levels. TG concentration ( $n=6$ mice per group) was measured by a TG Colorimetric Assay Kit (Cayman Chemical Company, Ann Arbor, MI, USA).

\section{Tissue collection and sample preparation}

The mice ( $n=4$ mice per group) were anesthetized with Zoletil (Virbac Laboratories) and then transcardially perfused with heparinized saline and $4 \%$ paraformaldehyde. Six hours after postfixing, the livers were processed for paraffin embedding and sectioned $(5 \mu \mathrm{m})$. Liver sections were stained with hematoxylin and eosin $(H \& E)$ and visualized under a BX51 light microscope (Olympus, Tokyo, Japan).

\section{Oil Red $O$ and Sirius Red staining}

Frozen liver sections $(5 \mu \mathrm{m})$ were stained for $10 \mathrm{~min}$ with $0.5 \%$ Oil Red O (Sigma-Aldrich, St. Louis, MO, USA), washed, and then counterstained for $45 \mathrm{sec}$ with Mayer's hematoxylin (Sigma-Aldrich), as previously described. ${ }^{16}$ To determine hepatic collagen accumulation, deparaffinized liver sections were stained with Weigert's hematoxylin (Sigma-Aldrich) for $8 \mathrm{~min}$, washed, restained with Sirius Red (Sigma-Aldrich) for $1 \mathrm{~h}$, and washed. The sections were visualized under a BX51 light microscope (Olympus).

\section{Western blot analysis}

Frozen liver tissue ( $n=6$ mice per group) was homogenized in a lysis buffer (15 mM HEPES [pH 7.9], $0.25 \mathrm{M}$ sucrose, $60 \mathrm{mM} \mathrm{KCl}, 10 \mathrm{mM} \mathrm{NaCl}, 1 \mathrm{mM}$ ethylene glycol tetraacetic acid, $1 \mathrm{mM}$ phenylmethylsulfonyl fluoride, and $2 \mathrm{mM} \mathrm{NaF}$ ). The primary antibodies used are listed in Sup- plementary Table S1 (Supplementary Data are available online at www.liebertpub.com/jmf). The $\beta$-actin and p84 were used as internal controls for cytosolic and nuclear extracts, respectively. Proteins were detected using enhanced chemiluminescence substrates (Pierce, Rockford, IL, USA). The Multi-Gauge image analysis program (version 3.0; Fujifilm, Tokyo, Japan) was used for densitometry analysis.

\section{Statistics}

Differences between groups were determined with one-way ANOVA and subsequent Bonferroni post hoc analysis. Statistical analysis of the $\alpha$-glucosidase inhibition study was performed by general linear model procedure, and significance was determined by Duncan's multiple range tests. Values are expressed as the mean \pm standard error of the mean. $P$-values less than .05 were considered statistically significant.

\section{RESULTS}

\section{Bioactivity-guided fractionation of $A A$ and isolation of compounds}

Bioactivity-guided fractionation of the $80 \%$ aqueous ethanol extract led to the isolation of 11 compounds. The structural identification of these compounds was carried out by spectroscopic analyses. We used an ACQUITY BEH C18 column, as shown in a previous study, ${ }^{17}$ which has frequently been used to separate sesquiterpenes and flavonoids from AA. Among these compounds, rhamnetin, myricetin, and chrysosplenol D have high biological activity in the $\alpha$-glucosidase inhibitor assay. The extracted compounds of AA were rhamnetin, myricetin, and chrysosplenol D (Fig. 1A), which are members of the flavonoid class. The structural characteristics, including ${ }^{1} \mathrm{H}$ and ${ }^{13} \mathrm{C}$ NMR spectroscopic data, are detailed in Table 1 and Supplementary Tables S2-S4. To pursue the potential antiobesity activity of
A

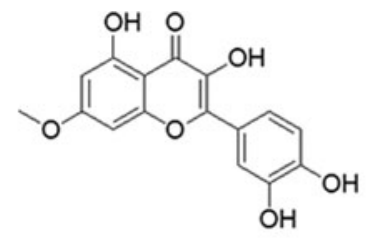

Rhamnetin

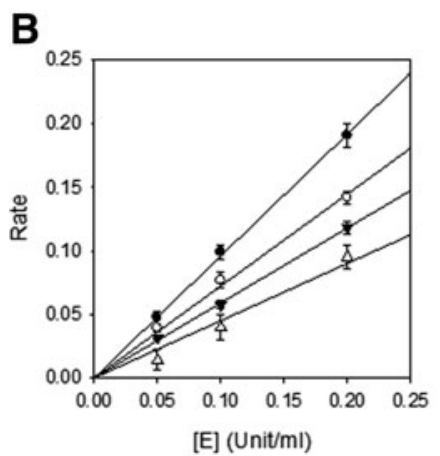

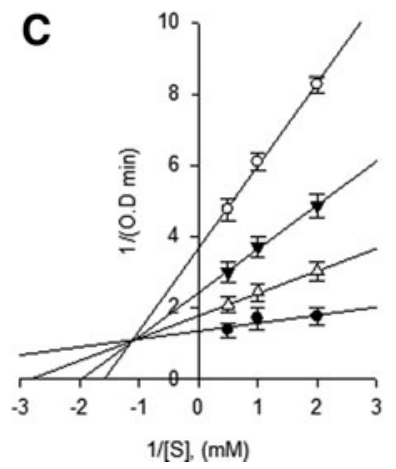

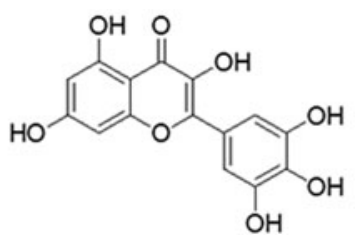

Myricetin

Chrysosplenol D

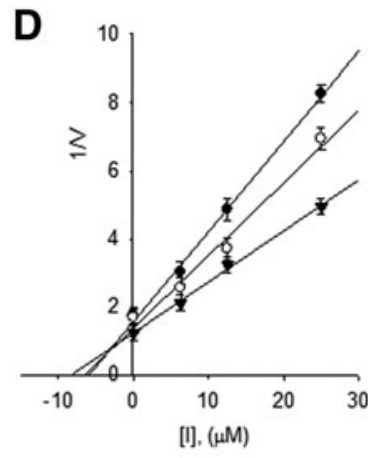

FIG. 1. (A) Chemical structure of components in AA extract: rhamnetin, myricetin, and chrysosplenol D. (B) The catalytic activity of $\alpha$-glucosidase as function of enzyme concentration at different concentrations of myricetin (, $0 \mu \mathrm{M} ; \bigcirc, 3.125 \mu \mathrm{M} ; \boldsymbol{\nabla}, 6.25 \mu \mathrm{M}$; and $\Delta, 12.5 \mu \mathrm{M})$. (C) LineweaverBurk plot analysis of the inhibition kinetics of $\alpha$-glucosidase by myricetin (, $0 \mu \mathrm{M} ; \bigcirc, 6.25 \mu \mathrm{M} ; \boldsymbol{\nabla}, 12.5 \mu \mathrm{M}$; and $\Delta, 25 \mu \mathrm{M})$; conditions were as follows: $0.15 \mathrm{mM}$ p-nitrophenyl- $\alpha$-Dglucopyranoside, 0.1 units of $\alpha$ glucosidase, and $50 \mathrm{mM}$ potassium phosphate buffer ( $\mathrm{pH} 6.8)$ at $37^{\circ} \mathrm{C}$. (D) Dixon plot analysis of the inhibition kinetics of $\alpha$-glucosidase by myricetin (○, $0.5 \mathrm{mM} ; \bigcirc, 1 \mathrm{mM}$; and $\boldsymbol{\nabla}$, $2 \mathrm{mM})$. AA, Artemisia annua. 
Table 1. NMR Spectroscopic Data (500 MHz, DMSO) for Compounds 1-3

\begin{tabular}{|c|c|c|c|c|c|c|}
\hline \multirow[b]{2}{*}{ No. of carbon } & \multicolumn{2}{|c|}{ 1. Rhamnetin } & \multicolumn{2}{|c|}{ 2. Myricetin } & \multicolumn{2}{|c|}{ 3. Chrysosplenol } \\
\hline & $\delta_{C}$ & $\delta_{H}(J$ in $H z)$ & $\delta_{C}$ & $\delta_{H}(J$ in $H z)$ & $\delta_{C}$ & $\delta_{H}(J$ in $H z)$ \\
\hline 2 & 147.1 & - & 147.3 & - & 152.1 & - \\
\hline 3 & 136.1 & - & 136.8 & - & 138.1 & - \\
\hline 4 & 175.8 & - & 176.2 & - & 178.7 & - \\
\hline 5 & 156.2 & - & 161.2 & - & 152.2 & - \\
\hline 6 & 97.4 & 6.34, d (1.97) & 98.6 & $6.19, \mathrm{~d}(2.1)$ & 132.0 & - \\
\hline 7 & 164.1 & - & 164.4 & - & 159.1 & - \\
\hline 8 & 91.7 & $6.72, \mathrm{~d}(1.97)$ & 93.7 & $6.38, \mathrm{~d}(2.1)$ & 91.7 & $6.86, \mathrm{~s}$ \\
\hline 9 & 160.5 & - & 156.5 & - & 156.5 & - \\
\hline 10 & 106.0 & - & 103.4 & - & 106.0 & - \\
\hline $1^{\prime}$ & 121.2 & - & 121.2 & - & 121.2 & - \\
\hline $2^{\prime}$ & 115.3 & $7.71, \mathrm{~d}(2.1)$ & 107.6 & $7.27, \mathrm{~s}$ & 116.2 & $7.61, \mathrm{~d}(2.1)$ \\
\hline $3^{\prime}$ & 145.8 & - & 146.2 & - & 145.8 & - \\
\hline $4^{\prime}$ & 146.9 & - & 146.2 & - & 149.4 & - \\
\hline $5^{\prime}$ & 115.2 & $6.90, \mathrm{~d}(8.5)$ & 146.2 & - & 116.0 & $6.92, \mathrm{~d}(8.5)$ \\
\hline $6^{\prime}$ & 121.0 & $7.51, \mathrm{dd}(8.5,2.1)$ & 107.6 & $7.27, \mathrm{~s}$ & 121.1 & $7.50, \mathrm{dd}(8.5,2.1)$ \\
\hline $3-\mathrm{OCH}_{3}$ & - & - & - & - & 60.1 & $3.80, \mathrm{~s}$ \\
\hline $6-\mathrm{OCH}_{3}$ & - & - & - & - & 60.5 & $3.73, \mathrm{~s}$ \\
\hline $7-\mathrm{OCH}_{3}$ & 56.9 & $3.89, \mathrm{~s}$ & - & - & 56.9 & $3.92, \mathrm{~s}$ \\
\hline
\end{tabular}

DMSO, dimethyl sulfoxide; NMR, nuclear magnetic resonance.

the three extracted flavonoids, ${ }^{18}$ we measured the $\alpha$ glucosidase inhibitory activities, as shown in Table 2 . The $\alpha$-glucosidase inhibitors reduce carbohydrate hydrolysis and glucose absorption, and thus, finding effective $\alpha$-glucosidase inhibitors is critical for treating type 2 diabetes. ${ }^{19,20} \mathrm{We}$ showed that myricetin had a significantly higher $\alpha$ glucosidase inhibition $\left(98.1 \pm 0.97 \%\right.$ inhibition; $\mathrm{IC}_{50}=$ $28.44 \pm 0.2 \mu \mathrm{M})$ than acarbose $(64.1 \pm 0.90 \%$ inhibition; $\left.\mathrm{IC}_{50}=324.83 \pm 0.3 \mu \mathrm{M}\right)$ at $500 \mu \mathrm{M}$ concentration.

We also identified the modes of inhibition using the Lineweaver-Burk and Dixon plots (Fig. 1B-D). The kinetic analysis of the inhibitor showed typical progress curves for reversible and mixed-type inhibition, with $\mathrm{K}_{\mathrm{i}}=12.2 \pm 0.7$ $\mu \mathrm{M}$. The results indicate that the AA extracts containing

Table 2. Inhibitory Effect of $\alpha$-Glucosidase of Separate COMPOUNDS FROM ARTEMISIA ANNUA L. LEAVES

\begin{tabular}{lcl}
\hline & \multicolumn{2}{c}{$\alpha$-Glucosidase inhibition } \\
\cline { 2 - 3 } Compound & Inhibition \%, & Type of inhibition \\
& $(500 \mu M)^{a, b}$ & $K_{i}(\mu M)^{b, c}$ \\
\hline Chrysosplenol D & $14.8 \pm 0.31$ & NT \\
Rhamnetin & $6.9 \pm 0.41$ & NT \\
Myricetin & $98.1 \pm 0.97$ & Mixed $(12.2 \pm 0.7)$ \\
& $\left(\mathrm{IC}_{50} \mu \mathrm{M}, 28.44 \pm 0.2\right)$ & \\
Acarbose & $64.1 \pm 0.90$ & $\mathrm{NT}$ \\
& $\left(\mathrm{IC}_{50} \mu \mathrm{M}, 324.83 \pm 0.3\right)$ & \\
\hline
\end{tabular}

\footnotetext{
${ }^{\mathrm{a}} \mathrm{All}$ compounds were examined in a set of experiments repeated three times; $\mathrm{IC}_{50}$ values of compounds represent the concentration that caused $50 \%$ enzyme activity loss.

${ }^{b}$ Each value is mean $\pm \operatorname{SEM}(n=3)$.

${ }^{\mathrm{c}}$ Values of inhibition constant.

SEM, standard error of the mean; NT, not tested.
}

myricetin have a potent $\alpha$-glucosidase activity and beneficial effects on obesity-induced metabolic disorders. Here, we investigated the AA effect in HFD-fed mice, with a particular focus on obesity-induced fatty liver disease.

\section{Effects of AA extract on body weight and blood glucose in HFD-fed mice}

To examine the antiobesity effect of AA extract on the HFD-fed mice, we measured body weights during the 12 weeks of extract administration. Figure $2 \mathrm{~A}$ shows that the body weight of HFD-fed mice increased compared to that of ND-fed mice, whereas the body weight of HFD-fed mice was slightly reduced by AA extract after 12 weeks. To determine the effect of AA administration on blood glucose levels in HFD-fed mice, we measured fasting blood glucose levels every 4 weeks. After 12 weeks, we observed a significant decrease in blood glucose levels in HFD+AA-fed mice compared with the HFD-fed mice (Fig. 2B). In addition, we found that the body and liver weights in HFD-fed mice were also reduced by AA administration (Fig. 2C, D).

\section{Effects of AA extract on insulin resistance in HFD-fed mice}

To determine whether AA extract improves insulin sensitivity in HFD-induced obesity, we performed the GTT and ITT (Fig. 3A-D). The HFD+AA-fed mice showed a significant reduction in the blood glucose levels during the GTT compared with the HFD-fed mice (Fig. 3A, B). Administration of AA extract also improved glucose tolerance upon insulin injection, and HFD+AA-fed mice exhibited reduced glucose levels during the ITT (Fig. 3C, D). Hyperinsulinemia in the HFD-fed mice was significantly reduced by the administration of AA extract (Fig. 3E). To 

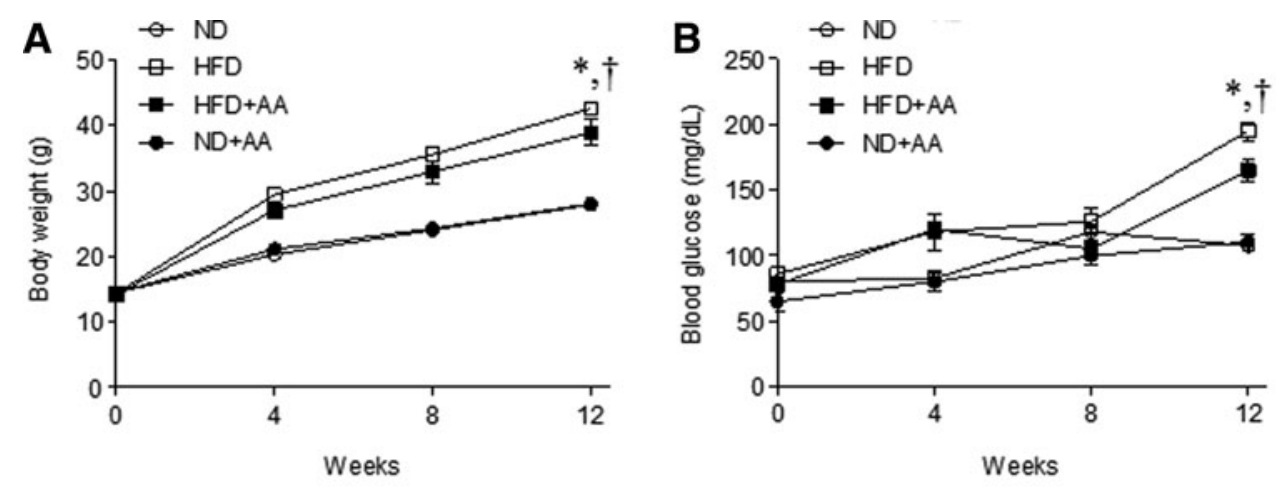

FIG. 2. Effects of AA extract on body weight and blood glucose in HFD-fed mice. Body weight (A) and fasting blood glucose (B) for 12 weeks of each treatment. Body weight (C) and liver weight (D) before sacrifice. Data ( $n=6$ mice per group) are presented as the mean \pm SEM. $* P<.05$ for ND-fed mice versus HFD-fed mice. ${ }^{\dagger} P<.05$ for HFD-fed mice versus

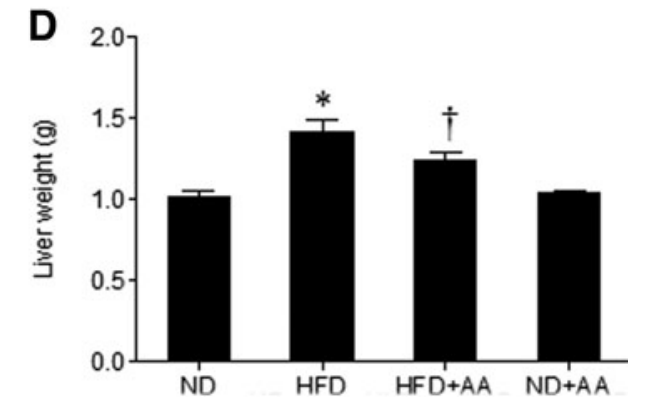
HFD+AA-fed mice. HFD, high-fat diet; ND, normal diet; SEM, standard error of the mean.

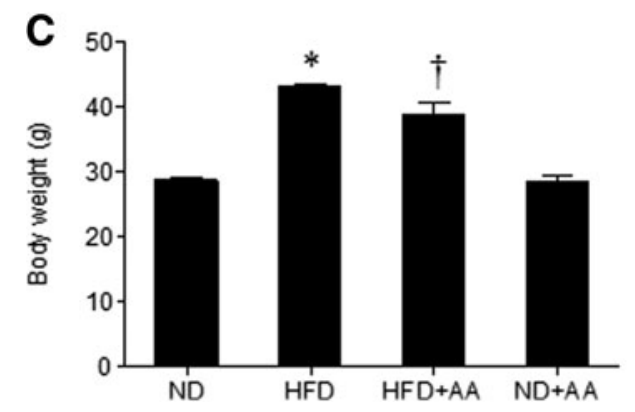

investigate whether AA extract affected hepatic function in the HFD-fed mice, we measured serum levels of the hepatic enzymes AST and ALT (Fig. 3F, G). AST and ALT concentrations were higher in the HFD-fed mice than in the ND-fed mice and decreased significantly in the HFD+AAfed mice. Total serum cholesterol in the HFD-fed mice was also significantly reduced after AA extract administration (Fig. 3H).

\section{Effect of $A A$ extract on hepatic steatosis in HFD-fed mice}

$\mathrm{H} \& \mathrm{E}$ and Oil Red $\mathrm{O}$ staining showed that the hepatocytes of the HFD-fed mice were filled with many lipid droplets but were decreased in HFD+AA-fed mice (Fig. 4A). The quantification of Oil Red O-positive area (\%) showed a significant decrease with AA extract administration
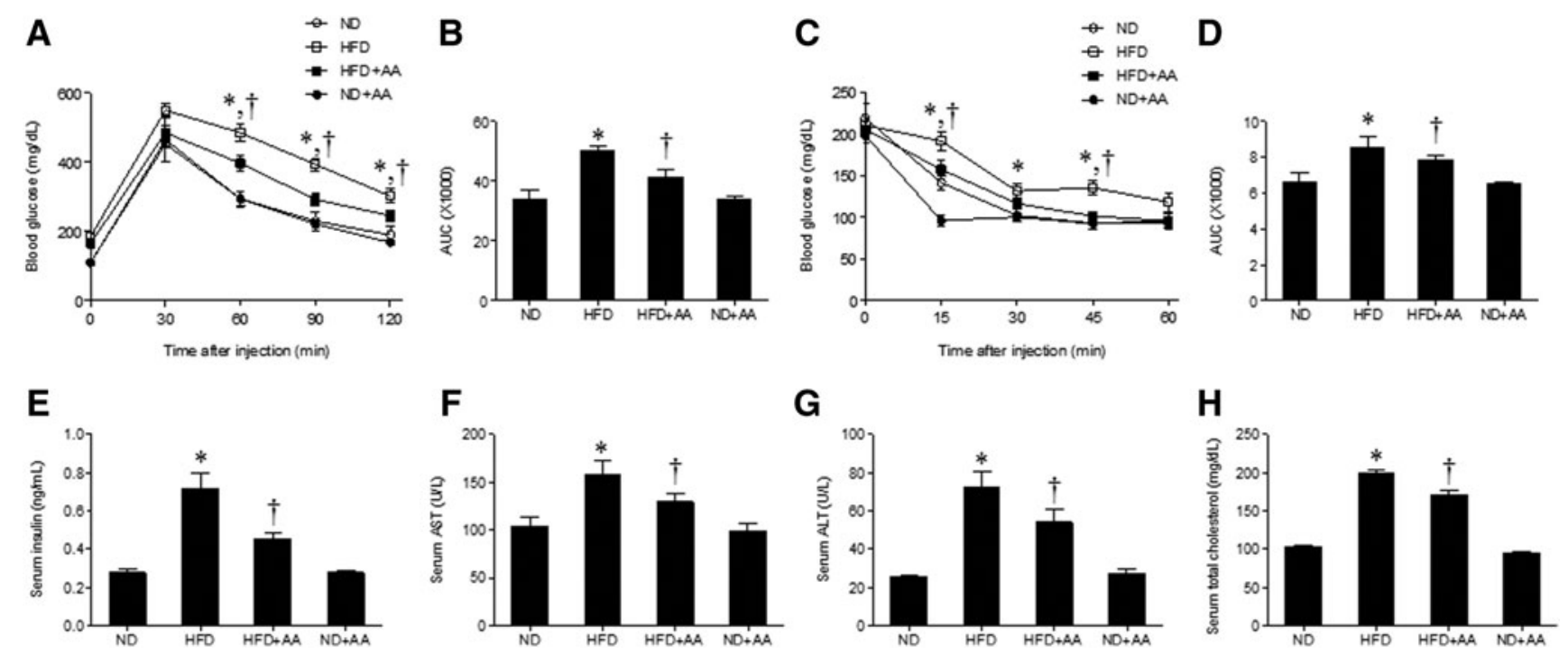

FIG. 3. Effects of AA extract on blood glucose, serum insulin, and metabolic parameters in HFD-fed mice. (A) Blood glucose levels after injection of D-glucose $(2 \mathrm{~g} / \mathrm{kg})$. (B) Area under the curve (AUC) for glucose tolerance test. (C) Blood glucose levels after insulin treatment $(0.75 \mathrm{U} / \mathrm{kg})$. (D) AUC for insulin tolerance test. Serum insulin (E), aspartate aminotransferase (F), alanine aminotransferase (G), and total cholesterol (H) levels. Data ( $n=6$ mice per group) are presented as the mean \pm SEM. $* P<.05$ for ND-fed mice versus HFD-fed mice. ${ }^{\dagger} P<.05$ for HFD-fed mice versus HFD+AA-fed mice. 
A
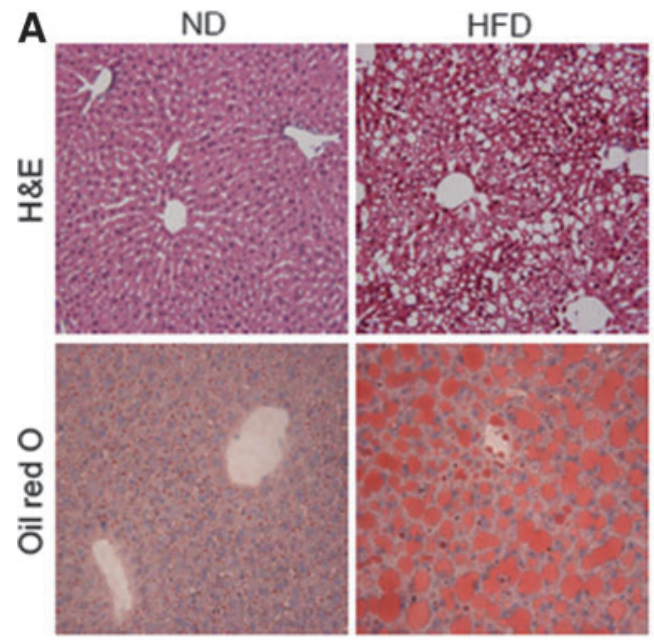

B

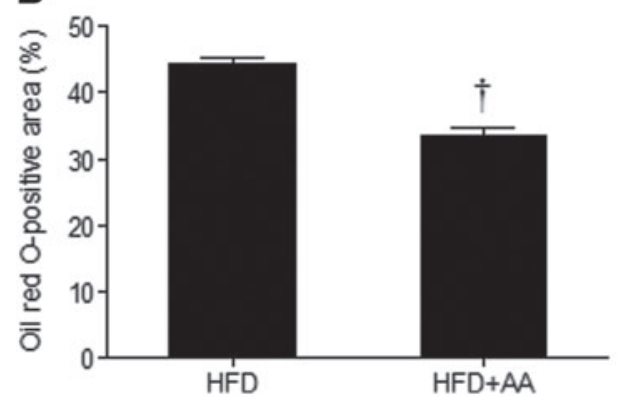

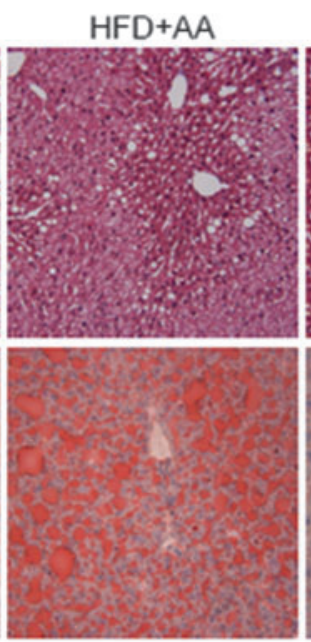

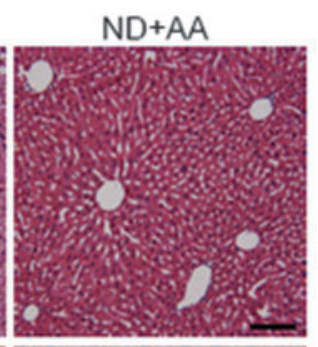

C

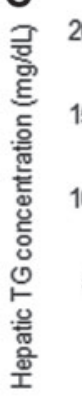

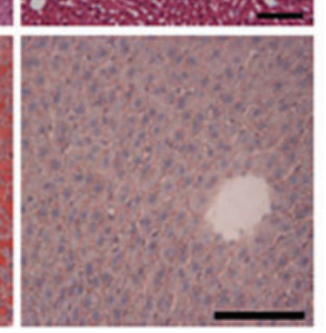

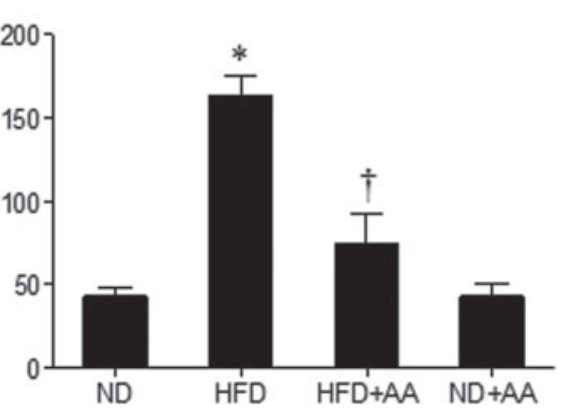

FIG. 4. Effect of AA extract on hepatic steatosis in HFD-fed mice. (A) Representative microphotographs of hematoxylin and eosin and Oil Red $\mathrm{O}$ staining. Scale bar $=100 \mu \mathrm{m}$. (B) The percentage of Oil Red O-positive areas. (C) Concentration of hepatic triglycerides. Data ( $n=6$ mice per group) are presented as the mean \pm SEM. $* P<.05$ for ND-fed mice versus HFDfed mice. ${ }^{\dagger} P<.05$ for HFD-fed mice versus HFD+AA-fed mice. Color images available online at www.liebertpub $. \mathrm{com} / \mathrm{jmf}$
(Fig. 4B). Consistently, the increased hepatic TG concentration in the HFD-fed mice was dramatically decreased in the livers of HFD+AA-fed mice, as shown by TG colorimetric assay (Fig. 4C).

\section{Effect of AA extract on hepatic lipogenesis in HFD-fed mice}

To investigate the effect of AA extract on hepatic lipogenesis, we examined the expression of p-ACC, ChREBP, and SREBP1, which are the key regulators of lipid synthesis. Hepatic p-ACC (an inactive form) level was significantly lower in the HFD-fed mice and significantly higher in the HFD+AA-fed mice (Fig. 5A), which is correlated with the changes in hepatic TG levels (Fig. 4C). HFD-induced nuclear ChREBP and SREBP1 expression was significantly decreased by AA extract administration (Fig. 5B, C).

\section{Effect of AA extract on hepatic inflammation and fibrogenesis in HFD-fed mice}

We found that HMGB1 and RAGE expression was higher in the HFD-fed mice than in the ND-fed mice, and AA extract administration significantly decreased HMGB1
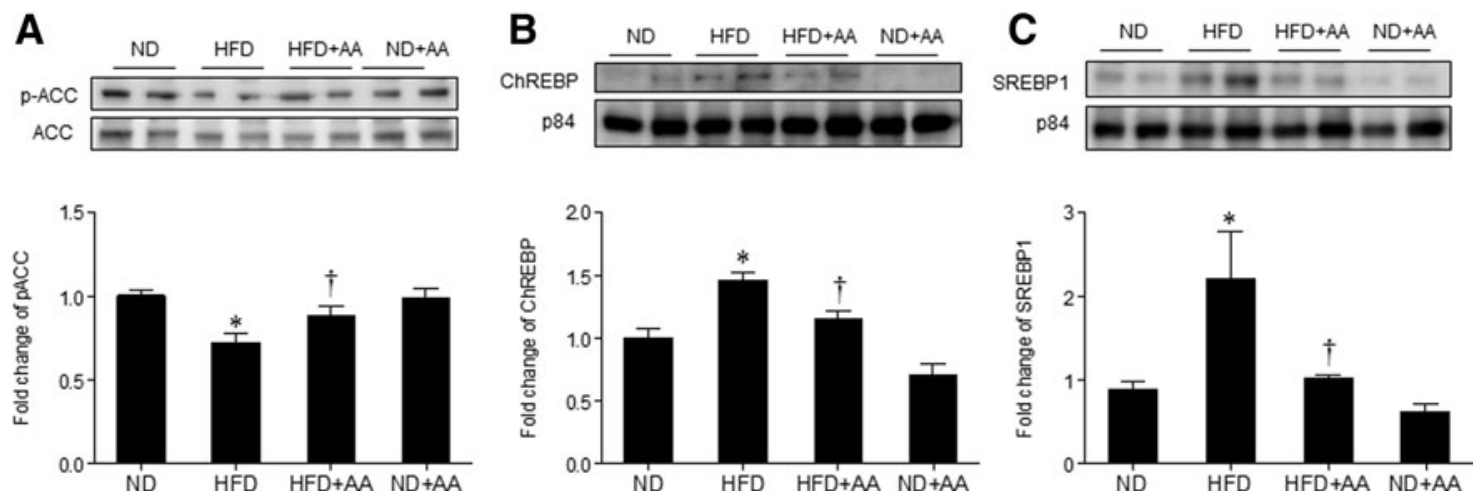

FIG. 5. Effect of AA extract on hepatic lipogenesis in HFD-fed mice. (A) Western blotting of hepatic p-ACC and ACC and quantification of p-ACC/ACC. (B) Western blotting of nuclear ChREBP and p84 in the liver and quantification of ChREBP/p84. (C) Western blotting of nuclear SREBP1 in the liver and quantification of SREBP/p84. Data ( $n=6$ mice per group) are presented as the mean \pm SEM. $* P<.05$ for ND-fed mice versus HFD-fed mice. ${ }^{\dagger} P<.05$ for HFD-fed mice versus HFD+AA-fed mice. ACC, acetyl-CoA carboxylase; ChREBP, carbohydrate-responsive element-binding protein; SREBP, sterol regulatory element-binding protein. 

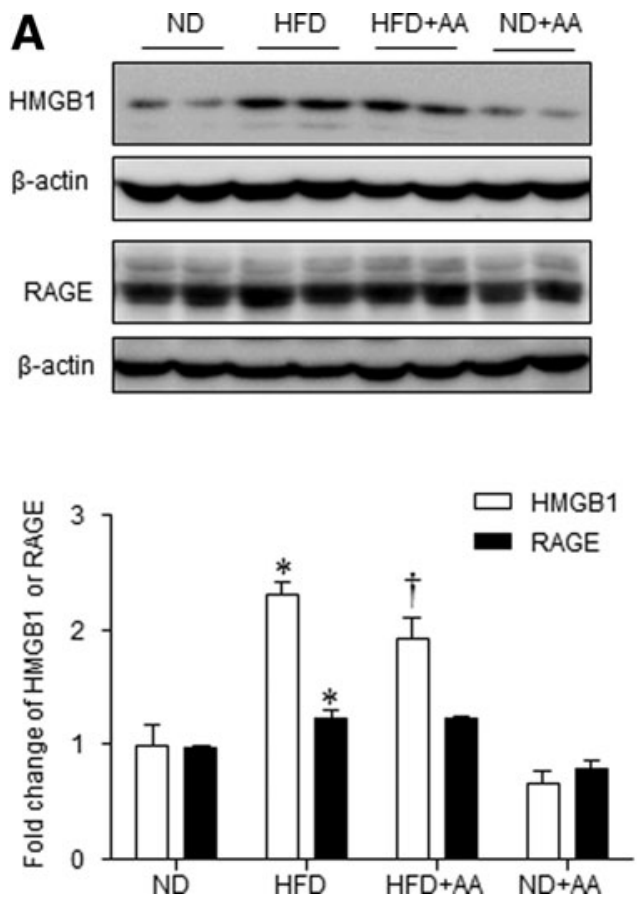
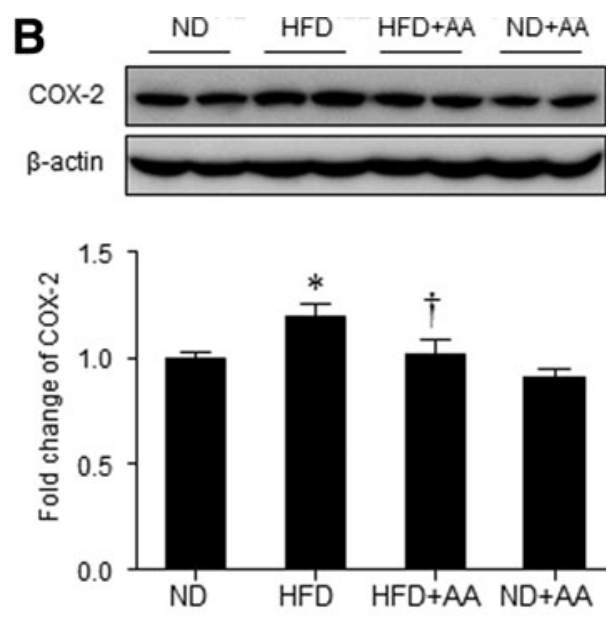

FIG. 6. Effects of AA extract on hepatic inflammation in HFD-fed mice. (A) Western blotting of hepatic HMGB1 and RAGE and quantification of HMGB $1 / \beta$ actin or RAGE/ $\beta$-actin. (B) Western blotting of hepatic COX-2 and quantification of $\mathrm{COX}-2 / \beta$-actin. Data $(n=6$ mice per group) are presented as the mean \pm SEM. $* P<.05$ for ND-fed mice versus HFD-fed mice. ${ }^{\dagger} P<.05$ for HFDfed mice versus HFD+AA-fed mice. COX-2, cyclooxygenase-2; HMGB, high-mobility group box. expression (Fig. 6A). However, RAGE expression in the HFD-fed mice was not significantly decreased by AA extract administration. Hepatic cyclooxygenase-2 (COX-2) expression was also higher in the HFD-fed mice than in the ND-fed mice; the COX-2 levels were also decreased significantly by AA extract administration (Fig. 6B). Next, the effect of AA extract on the HFD-induced hepatic fibrogenesis was evaluated by western blot analysis of TGF- $\beta 1$ and CTGF (Fig. 7A, B). Hepatic TGF- $\beta 1$ and CTGF expression was considerably higher in the HFD-fed mice than in the ND-fed mice; the TGF- $\beta 1$ and CTGF levels were also decreased by AA extract administration. In addition, HFD+AA-fed mice displayed less Sirius Red staining and hepatic collagen levels compared with HFD-fed mice (Fig. 7C, D).

\section{DISCUSSION}

AA is a well-known source of the antimalarial drug artemisinin and has been used for the treatment of fevers and malaria for centuries in traditional and herbal medicine. Artemisinin was isolated from AA in 1971 and is currently the most powerful antimalarial drug ever developed ${ }^{21}$ and known to have anti-inflammatory and antioxidant activities. ${ }^{3,4}$ AA has also been used for the treatment of diabetes in herbal medicine; however, its molecular mechanism of antidiabetic function has not been clearly investigated. Obesity is associated with a chronic low-level inflammation; HFDinduced hyperglycemia increases endoplasmic reticulum stress and oxidative stress that activate inflammatory cytokines. $^{22}$ Thus, we hypothesized that the anti-inflammatory and antioxidant activities in AA extract may prevent obesity-induced insulin resistance and hepatic steatosis in
HFD-fed mice. In fact, myricetin, one of the three flavonoids present in AA extract, possesses antioxidative properties and improves insulin sensitivity. ${ }^{18,23}$ Dietary myricetin also decreases the body weight of HFD-fed rats. ${ }^{24}$ In this study, we showed that the administration of AA extract significantly reduced obesity and insulin resistance in HFD-fed mice. We also showed in vitro that AA dose dependently inhibited $\alpha$-glucosidase activity even more effectively than acarbose, a known antidiabetic drug. We found that serum hepatic enzymes and TG levels were reduced by AA extract administration in HFD-fed mice, suggesting that AA extract plays a role in reducing hepatic dysfunction and fat accumulation observed in these animals.

NAFLD is one of the most common liver conditions and is associated with insulin resistance and diabetes. ${ }^{25}$ The liver of obese rodents, such as the ob/ob or lipodystrophic mice, is characterized by severe hepatic insulin resistance resulting in glucose overproduction and hyperglycemia and overinduction of hepatic SREBP1. ${ }^{26}$ Studies in mice have revealed that ChREBP is a crucial modulator of hepatic fatty acid content through its transcriptional control of lipogenic genes. ${ }^{10,27}$ ChREBP overexpression in the liver of HFD-fed mice leads to the development of hepatic steatosis. ${ }^{10}$ Consistently, we showed that the hepatic nuclear expression of SREBP1 and ChREBP was significantly higher in the HFDfed mice, whereas AA extract administration markedly decreased the expression. These results indicate that the HFD+AA-fed mice were protected from hepatic steatosis induced by lipogenesis through nuclear SREBP1 and ChREBP. Administration of AA extract decreased nuclear levels of SREBP1 and ChREBP and increased the phosphorylation of $\mathrm{ACC}$, resulting in decreased hepatic de novo lipogenesis and lipid accumulation. 

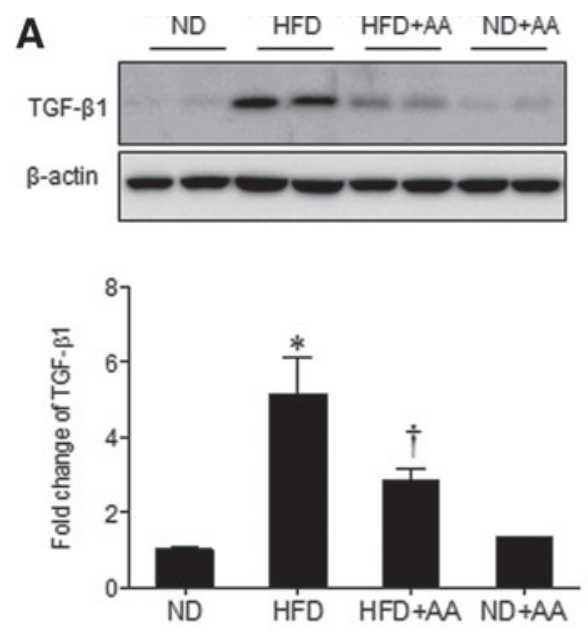

C
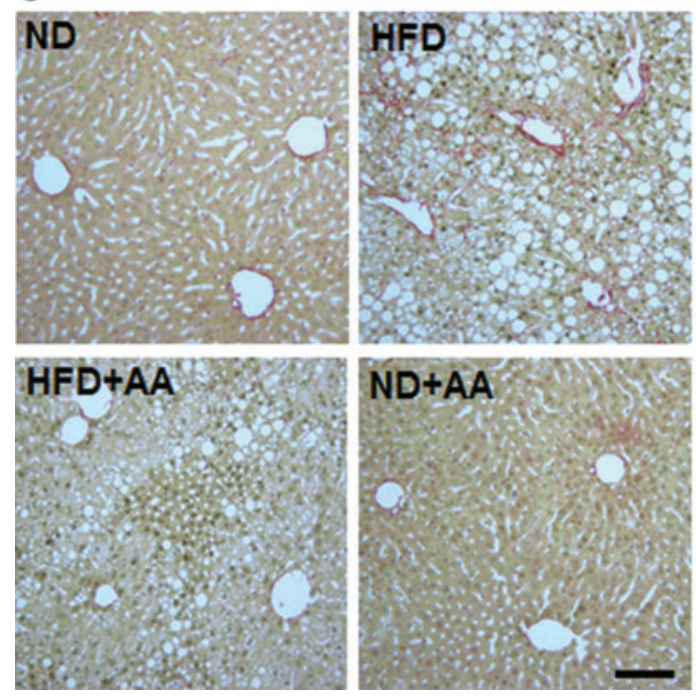
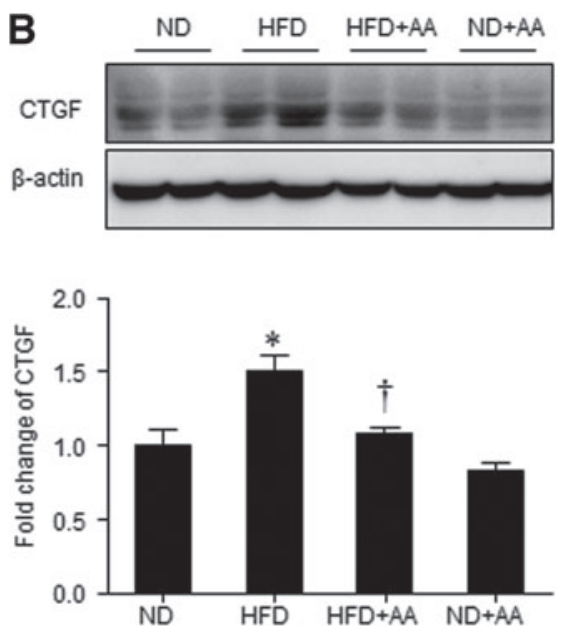

D

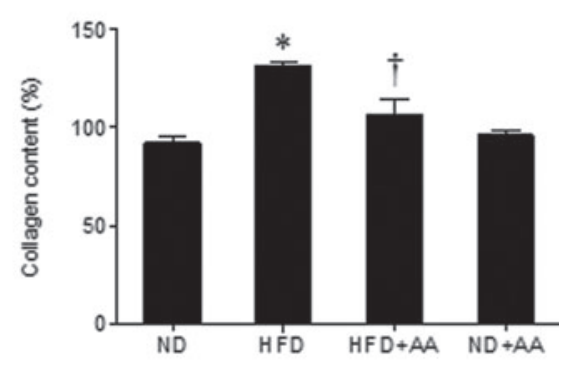

FIG. 7. Effects of AA extract on hepatic fibrogenesis in HFD-fed mice. (A) Western blotting of hepatic TGF- $\beta 1$ and quantification of TGF- $\beta 1 / \beta$-actin. (B) Western blotting of hepatic CTGF and quantification of $\mathrm{CTGF} / \beta$-actin. (C) Representative microphotographs of Sirius Red staining. Scale bar $=100 \mu \mathrm{m}$. (D) Hepatic collagen levels. Data $(n=6$ mice per group) are presented as the mean \pm SEM. $* P<.05$ for ND-fed mice versus HFD-fed mice. ${ }^{\dagger} P<.05$ for HFD-fed mice versus HFD+AA-fed mice. CTGF, connective tissue growth factor; TGF- $\beta 1$, transforming growth factor- $\beta 1$. Color images available online at www.liebertpub.com/jmf
ChREBP is involved in various processes of glucose and lipid metabolism, which are differentially regulated by genetic, dietary, or environmental factors. ${ }^{10}$ The activity of ChREBP is regulated by phosphorylation at multiple sites under high-glucose conditions, suggesting that ChREBP provides a causal relationship between hyperglycemia and lipogenesis. ${ }^{28}$ The adenoviral shRNA-mediated inhibition of ChREBP improves hepatic steatosis and insulin resistance in genetically obese (ob/ob) mice, ${ }^{29}$ and ChREBPdeficient ob/ob mice are significantly lower in both lipogenic and gluconeogenic enzymes. ${ }^{30}$ However, adenoviral induced ChREBP-overexpressing mice fed an HFD have better glucose tolerance, despite increased hepatic steatosis; furthermore, ChREBP expression is positively related to the degree of steatosis and inversely to insulin resistance in patients with NASH. ${ }^{31}$ Our data showed that ChREBP expression was increased in the HFD-fed mice that showed hepatic steatosis and insulin resistance and that AA extract administration decreased ChREBP expression and improved both hepatic steatosis and insulin resistance. Further study is re- quired to specify the effective components in AA extract to regulate ChREBP signaling and the differential effects on glucose and lipid metabolism in obesity.

Hepatic fibrosis can result from a number of chronic liver diseases. ${ }^{7}$ In a rat model of hepatic fibrosis, the level of HMGB1 is upregulated, and the expression is closely correlated with collagen deposition, whereas the suppression of HMGB 1 expression by small interfering RNA significantly inhibits collagen synthesis in hepatic stellate cells. ${ }^{32} \mathrm{COX}-2$ is involved in the development of NASH; the products of lipid peroxidation can mediate inflammatory cell recruitment by activating COX-2. ${ }^{33} \mathrm{COX}-2$-mediated adipose tissue inflammation is crucial to the development of insulin resistance and fatty liver in HFD-induced obese rats. ${ }^{34}$ Here, we also showed that HMGB1 and COX-2 levels, as well as collagen deposition, were markedly elevated in the HFD-fed mice, and we further showed that they were decreased by dietary AA extract.

CTGF is a profibrogenic cytokine that acts downstream of the fibrogenic master cytokine TGF- $\beta 1$ and synergizes the 
activity. Upregulated expression of CTGF is commonly observed in many types of fibrotic tissues. ${ }^{35}$ Our results showed that HFD-fed mice had increased CTGF expression, which was correlated with increased expression of TGF- $\beta 1$. The administration of AA extract decreased CTGF and TGF- $\beta 1$ hepatic expression in the HFD-fed mice. AA extract markedly decreased hepatic collagen deposition and fibrosis in the HFD-fed mice, suggesting that the antiinflammatory effect of AA may contribute to protection against fibrotic progression.

In conclusion, our results indicate that AA extract administration may prevent the development of hepatic fibrosis by reducing lipid accumulation and inflammation in the liver. AA extract may be beneficial for the prevention of obesity-induced metabolic syndromes, illustrating the importance of further studies to determine key molecular players to mediate the antiobesity effect of AA.

\section{ACKNOWLEDGMENTS}

This work was supported by grants from the Projects of Grant (No. S2148343) from Small and Medium Business Administration and the Basic Science Research Program through the National Research Foundation of Korea (No. 2014R1A2A1A11049588).

\section{AUTHOR DISCLOSURE STATEMENT}

No competing financial interests exist.

\section{REFERENCES}

1. Räth K, Taxis K, Walz G, Gleiter CH, Li SM, Heide L: Pharmacokinetic study of artemisinin after oral intake of a traditional preparation of Artemisia annua L. (annual wormwood). Am J Trop Med Hyg 2004;70:128-132.

2. Ho WE, Peh HY, Chan TK, Wong WS: Artemisinins: Pharmacological actions beyond anti-malarial. Pharmacol Ther 2014;142:126-139.

3. Kim MH, Seo JY, Liu KH, Kim JS: Protective effect of Artemisia annua $L$. extract against galactose-induced oxidative stress in mice. PLoS One 2014;9:e101486.

4. Zheng GQ: Cytotoxic terpenoids and flavonoids from Artemisia annua. Planta Med 1994;60:54-57.

5. Sun B, Karin M: Obesity, inflammation, and liver cancer. $J$ Hepatol 2012;56:704-713.

6. Postic C, Girard J: The role of the lipogenic pathway in the development of hepatic steatosis. Diabetes Metab 2008;34:643648 .

7. Abelmalek MF, Diehl AM: Nonalcoholic fatty liver disease as a complication of insulin resistance. Med Clin North Am 2007;91: 1125-1149.

8. Tu K, Zheng X, Yin G, Zan X, Yao Y, Liu Q: Evaluation of Fbxw7 expression and its correlation with expression of SREBP-1 in a mouse model of NAFLD. Mol Med Rep 2012;6: 525-530.

9. Dentin R, Girard J, Postic C: Carbohydrate responsive element binding protein (ChREBP) and sterol regulatory element binding protein-1c (SREBP-1c): Two key regulators of glucose metabolism and lipid synthesis in liver. Biochimie 2005;87:81-86.
10. Filhoulaud G, Guilmeau S, Dentin R, Girard J, Postic C: Novel insights into ChREBP regulation and function. Trends Endocrinol Metab 2013;24:257-268.

11. Foufelle F, Ferré P: New perspectives in the regulation of hepatic glycolytic and lipogenic genes by insulin and glucose: A role for the transcription factor sterol regulatory element binding protein1c. Biochem J 2002;366:377-391.

12. Kamari Y, Shaish A, Vax E, et al.: Lack of interleukin- $1 \alpha$ or interleukin- $1 \beta$ inhibits transformation of steatosis to steatohepatitis and liver fibrosis in hypercholesterolemic mice. J Hepatol 2011;55:1086-1094.

13. Chen R, Hou W, Zhang Q, Kang R, Fan XG, Tang D: Emerging role of high-mobility group box 1 (HMGB1) in liver diseases. Mol Med 2013;19:357-366.

14. Chiang DJ, Pritchard MT, Nagy LE: Obesity, diabetes mellitus, and liver fibrosis. Am J Physiol Gastrointest Liver Physiol 2011; 300:G697-G702.

15. Matsui T, Ueda T, Oki T, Sugita K, Terahara N, Matsumoto K: $\alpha$-Glucosidase inhibitory action of natural acylated anthocyanins. 1. Survey of natural pigments with potent inhibitory activity. $J$ Agric Food Chem 2001;49:1948-1951.

16. Jeon BT, Heo RW, Shin HJ, et al.: Attenuation by a Vigna nakashimae extract of nonalcoholic fatty liver disease in high-fat diet-fed mice. Biosci Biotechnol Biochem 2014;78:482-489.

17. Alexei L, Eba A, Benhilda NM, et al.: Integrating medicinal plants extraction into a high-value biorefinery: An example of Artemisia annua L. C R Chim 2014;17:232-241.

18. Choi HN, Kang MJ, Lee SJ, Kim JI: Ameliorative effect of myricetin on insulin resistance in mice fed a high-fat, highsucrose diet. Nutr Res Pract 2014;8:544-549.

19. Huang GJ, Hsieh WT, Chang HY, Huang SS, Lin YC, Kuo YH: $\alpha$-Glucosidase and aldose reductase inhibitory activities from the fruiting body of Phellinus merrillii. J Agric Food Chem 2011;59: 5702-5706.

20. Kumar S, Narwal S, Kumar V, Prakash O: $\alpha$-Glucosidase inhibitors from plants: A natural approach to treat diabetes. Pharmacogn Rev 2011;5:19-29.

21. Willcox ML, Burton S, Oyweka R, Namyalo R, Challand S, Lindsey K: Evaluation and pharmacovigilance of projects promoting cultivation and local use of Artemisia annua for malaria. Malar J 2011;10:84.

22. Wellen KE, Hotamisligil GS: Inflammation, stress, and diabetes. J Clin Invest 2005;115:1111-1119.

23. Liu IM, Tzeng TF, Liou SS, Lan TW: Improvement of insulin sensitivity in obese Zucker rats by myricetin extracted from Abelmoschus moschatus. Planta Med 2007;73:1054-1060.

24. Chang CJ, Tzeng TF, Liou SS, Chang YS, Liu IM: Myricetin increases hepatic peroxisome proliferator-activated receptor $\alpha$ protein expression and decreases plasma lipids and adiposity in rats. Evid Based Complement Alternat Med 2012;2012:787152.

25. Sakurai M, Takamura T, Ota T, et al.: Liver steatosis, but not fibrosis, is associated with insulin resistance in nonalcoholic fatty liver disease. J. Gastroenterol 2007;42:312-317.

26. Ferré P, Foufelle F: Hepatic steatosis: A role for de novo lipogenesis and the transcription factor SREBP-1c. Diabetes Obes Metab 2010;12:83-92.

27. Iizuka K, Bruick RK, Liang G, Horton JD, Uyeda K: Deficiency of carbohydrate response element-binding protein (ChREBP) reduces lipogenesis as well as glycolysis. Proc Natl Acad Sci USA 2004;101:7281-7286. 
28. Tsatsos NG, Davies MN, O'Callaghan BL, Towle HC: Identification and function of phosphorylation in the glucose-regulated transcription factor ChREBP. Biochem J 2008;411:261-270.

29. Dentin R, Benhamed F, Hainaylt, et al.: Liver-specific inhibition of ChREBP improves hepatic steatosis and insulin resistance in ob/ob mice. Diabetes 2006;55:2159-2170.

30. Iizuka K, Miller B, Uyeda K: Deficiency of carbohydrateactivated transcription factor ChREBP prevents obesity and improves plasma glucose control in leptin-deficient (ob/ob) mice. Am J Physiol Endocrinol Metab 2006;291:E358-E364.

31. Benhamed F, Denechaud PD, Lemoine M, et al:: The lipogenic transcription factor ChREBP dissociates hepatic steatosis from insulin resistance in mice and humans. J Clin Invest 2012;122: 2176-2194.
32. Ge WS, Wu JX, Fan JG, Wang YJ, Chen YW: Inhibition of highmobility group box 1 expression by siRNA in rat hepatic stellate cells. World J. Gastroenterol 2011;17:4090-4098.

33. Chen J, Liu D, Bai Q, et al.: Celecoxib attenuates liver steatosis and inflammation in non-alcoholic steatohepatitis induced by high-fat diet in rats. Mol Med Rep 2011;4:811-816.

34. Hsieh PS, Jin JS, Chiang CF, Chan PC, Chen CH, Shih KC: COX-2-mediated inflammation in fat is crucial for obesity-linked insulin resistance and fatty liver. Obesity (Silver Spring) 2009; 17:1150-1157.

35. Lipson KE, Wong C, Teng Y, Spong S: CTGF is a central mediator of tissue remodeling and fibrosis and its inhibition can reverse the process of fibrosis. Fibrogenesis Tissue Repair 2012; 5:S24. 